\title{
A 3-axis accelerometer and strain sensor system for building integrity monitoring
}

\author{
J. Santana ${ }^{\mathrm{a}, *}$, R. van den Hoven ${ }^{\mathrm{a}}$, C. van Liempd ${ }^{\mathrm{a}}$, M. Colin ${ }^{\mathrm{b}}$, N. Saillen ${ }^{\mathrm{c}}$, D. Zonta $^{\mathrm{d}}$, D. Trapani ${ }^{\mathrm{d}}$, T. Torfs $^{\mathrm{e}}$, \\ C. Van Hoof ${ }^{a, e}$
}

a imec-Holst Centre, Eindhoven, The Netherlands

${ }^{\mathrm{b}} \mathrm{MEMSCAP}$, Grenoble, France

c Thermo Fisher Scientific, Enschede, The Netherlands

${ }^{\mathrm{d}}$ University of Trento, Italy

e IMEC, Leuven, Belgium

\section{A R T I C L E I N F O}

\section{Article history:}

Received 9 August 2011

Received in revised form

10 November 2011

Accepted 11 November 2011

Available online $\mathrm{xxx}$

\section{Keywords:}

Signal-to-Noise ratio

Linearity

Bandwidth

Sensitivity

Integrated number of pulses

Residual motion

\begin{abstract}
A B S T R A C T
An Ultra-Low-Power readout architecture for capacitive MEMS-based accelerometers and strain sensors is presented. The system can read both accelerometers and strain sensors in a half-bridge configuration. An accurate VerilogA model of the sensor was made to improve simulations. The gain of the system is controlled by integrating pulses from the excitation circuit allowing accurate control of the Signal-toNoise ratio. A Figure-of-Merit of $4.41 \times 10^{-20} \mathrm{~F} \sqrt{ }(\mathrm{W} / \mathrm{Hz})$ was achieved for a sensor range of $\pm 2.0 \mathrm{~g}$ and $\pm 20,000 \mu \varepsilon$ over a $100 \mathrm{~Hz}$ bandwidth. A minimum of $440 \mathrm{nW}$ power consumption was recorded. Residual motion artifacts are also cancelled by the system.
\end{abstract}

(C) 2011 Elsevier B.V. All rights reserved.

\section{Introduction}

An accurate sensing of signals requires innovative, flexible and power efficient readout architectures. In fields such as building integrity which use a wide range of capacitive MEMS/NEMS-based devices, such as accelerometers and strain sensors with different actuation voltages, sensitivities and resolutions, the specifications of the sensor and system are increasingly demanding. Resolutions of $1 \mathrm{mg}$ and $10 \mu \varepsilon$ are required for the accelerometer and strain sensor respectively and a range of $\pm 2.0 \mathrm{~g}$ and $\pm 20,000 \mu \varepsilon$ over a $100 \mathrm{~Hz}$ bandwidth to sense accelerations and stresses. This is essential for the assessment of structures during and after seismic events.

This work introduces a unique Ultra-Low-Power system which can be applied to MEMS-based comb finger capacitive accelerometers or strain sensors in a half-bridge configuration [1]. An accurate behavioral model of the sensor (in VerilogA language) was made in order to simulate the full system and improve its performance. In comparison with current modulation-demodulation readout techniques the gain of the system can be set by the number of integration pulses $N$ coming from the excitation voltage, optimizing

\footnotetext{
* Corresponding author. Tel.: +31 404020548; fax: +31 404020699.

E-mail address: juan.santana@imec-nl.nl (J. Santana).
}

then SNR and bandwidth with power. In addition the architecture suppresses motion artifacts [2,3] such as residual motion.

\section{Sensors}

The accelerometer sensor consists of 2 transverse comb finger structures ( $X$ and $Y$ axis) and, a pendulating one for the $Z$ axis (Fig. 1).

The sensors are made using a cap structure (Fig. 2), its purpose is twofold; it protects the MEMS structure and also forms the counter electrode for the $Z$ sensor. Innovative cap through connections were used. The cap itself is mounted to the MEMS wafer by using a metal-silicon eutectic bonding processes. The metal for the sealing ring is also used to realize the electrical connections between the cap and the MEMS wafer. The accelerometer was fabricated with a surface micro-machined process from a SOI wafer $85 \mu \mathrm{m}$ thick. The sensor has 78 fingers with a total sensitivity of $2.02 \mathrm{pF} / \mathrm{g}$. The $\mathrm{Z}$ sensor has an area of $2.17 \mathrm{~mm}^{2}$ per plate.

The main tradeoff in the design of the accelerometer is the sensitivity-bandwidth-linearity in all three axes, a challenge for the design given the different used structures.

The strain sensor is a longitudinal comb finger capacitor (Fig. 3). The sensor consists of three parts: the connector area (for connection to the readout), the flexible area (to sustain the strain and stress of the bars) and the sensor area (the active device). The strain sensor 


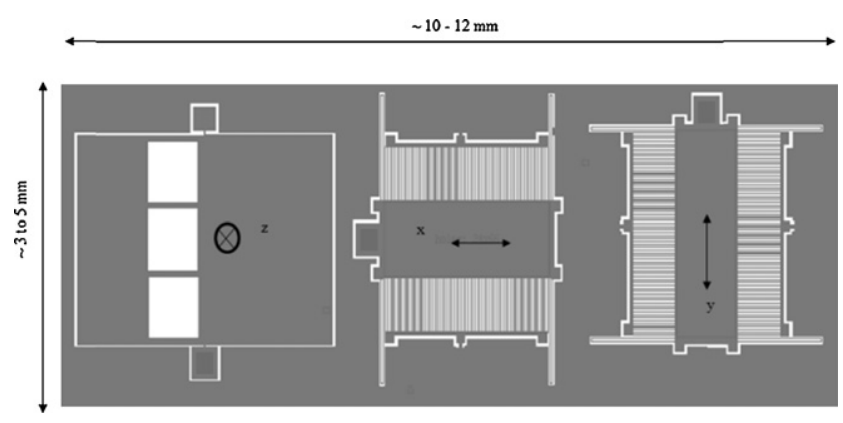

Fig. 1. 3-Axis accelerometer arrangement.

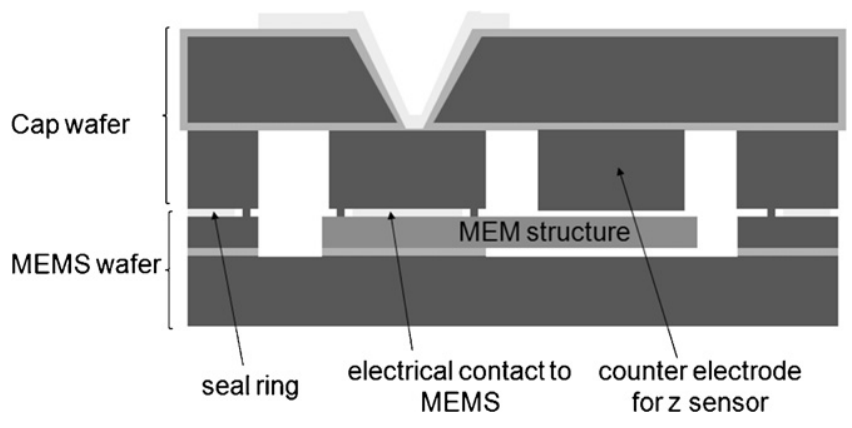

Fig. 2. Cross section of the accelerometer sensor.

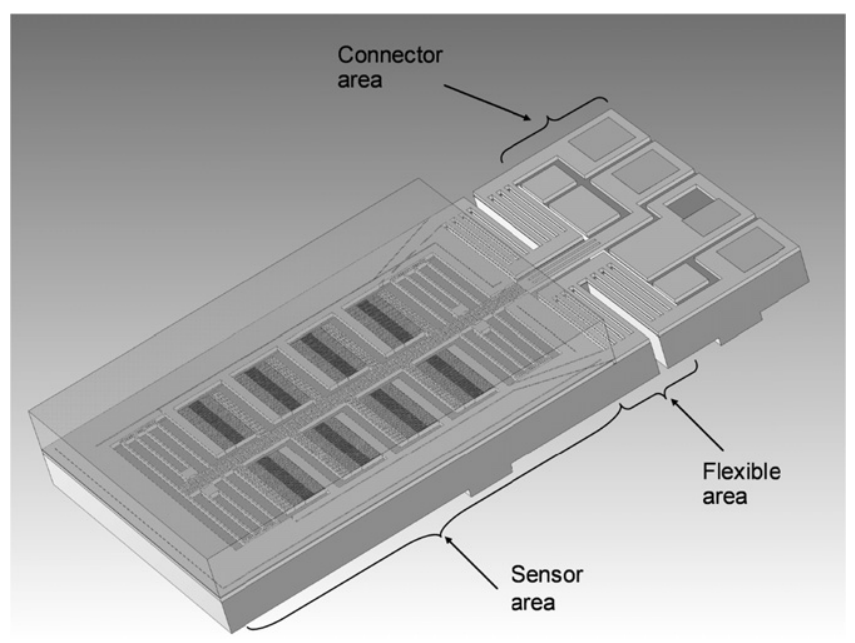

Fig. 3. 3D representation of the strain sensor.

fabrication uses a SOI wafer with a $500 \mu \mathrm{m}$ thick handle, $50 \mu \mathrm{m}$ thick fingers and $2 \mu \mathrm{m}$ thick oxide layer with 400 fingers in the sensor and it has a sensitivity of $0.133 \mathrm{fF} / \mu \varepsilon$.

Two anchors were etched-out of the surface to create the necessary clamps to attach the sensor to the rebar of a pillar. The fingers are protected with a borosilicate class cap. The specifications of the MEMS-based sensors used in this work are given in Table 1. The voltage vs. frequency response of the accelerometer as function of pressure is shown in Fig. 4. The plot shows the transition of the accelerometer from an over-damped system (at 820 Torr) to an under-damped system (at 3 Torr). The temperature dependence of the accelerometer and strain sensor is compensated by using a temperature sensor close to the accelerometer/strain sensor and using look up tables for calibration. No long term drift is expected because of the good aging properties of silicon in the cap wafer. Fine leaks could appear in the bonding layers which will make the
Table 1

Accelerometer and strain sensor specifications. The $X, Y$ axis sensors and $Z$ axis are given separate.

\begin{tabular}{lll}
\hline Parameter & Accelerometer & Strain sensor \\
\hline Basic & $20 \mathrm{pF}(X, Y)$ & $5.3 \mathrm{pF}$ \\
Capacitance & $20 \mathrm{pF}(Z)$ & \\
& $0.34 \mathrm{mg}(X, Y)$ & - \\
& $0.5 \mathrm{mg}(Z)$ & $3 \mathrm{~nm} / \mu \varepsilon$ \\
Mechanical sensitivity & $0.4-0.06 \mu \mathrm{m} / \mathrm{g}$ & $0.133 \mathrm{fF} / \mu \varepsilon$ \\
Electrical sensitivity & $0.23-2.02 \mathrm{pF} / \mathrm{g}(X, Y)$ & $56 \mathrm{kHz}$ \\
& $0.38-0.94 \mathrm{pF} / \mathrm{g}(Z)$ & \\
Resonant & $785-2080 \mathrm{~Hz}(X, Y)$ & - \\
Frequency & $780-1015(Z)$ & \\
Pull-in voltage & $2.3-5.4 \mathrm{~V}(X, Y)$ & $4 \mu \mathrm{m}$ \\
& $0.95-1.3 \mathrm{~V}(Z)$ & \\
Finger gap & $3 \mu \mathrm{m}$ & \\
\hline
\end{tabular}

pressure to rise inside the MEMS cavity but these issues are beyond the scope of the paper.

Fig. 5 shows the fabricated sensors and the readout ASIC. As mentioned in previous paragraphs the same readout ASIC is used for both the accelerometer and strain sensors, making this a costeffective solution when compared to other systems.

\section{Behavioral model of the sensor}

As mentioned in Section 1 an accurate model of the sensor is necessary in order to optimize the design of the readout. As the trend for low-power continues to require power saving systems an optimum trade-off between sensors and readout is necessary to reach such demanding expectations, therefore accurate models of the sensors which can be used along in the transistor-level simulations are required. Such models have been known for several years and in several forms, look-up tables, behavioral models, macro-models, etc. In this study a behavioral description language was chosen to model the sensor, namely VerilogA, mainly because it is a tool included in the used design environment.

A variable capacitor with three terminals was made to simulate the sensors; the third terminal simulates the displacement of the mass when an external force is applied to the accelerometer, such as that coming from an earthquake, or stress in the case of the stress sensor. In either case the model can be easily customized to each sensor, a parameterized model was developed so to be easily adapted to different requirements.

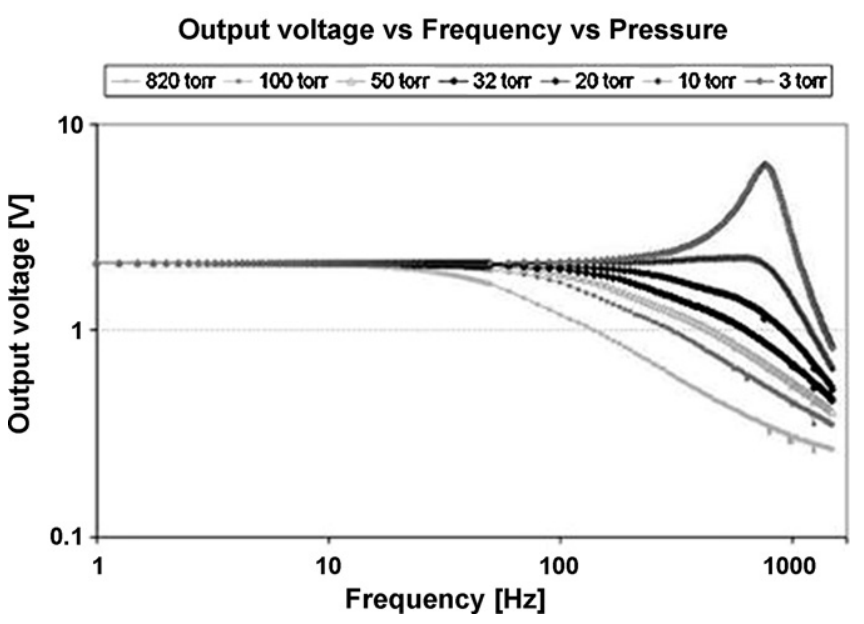

Fig. 4. Voltage vs. frequency response of the accelerometer as function of pressure 


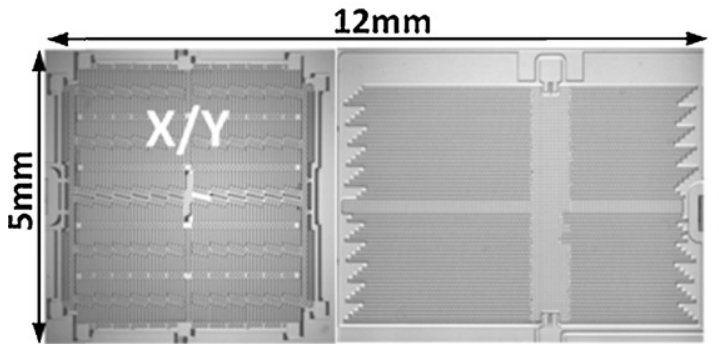

3-axis accelerometer

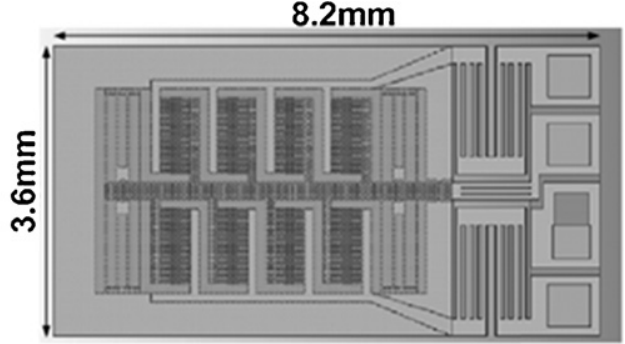

strain sensor

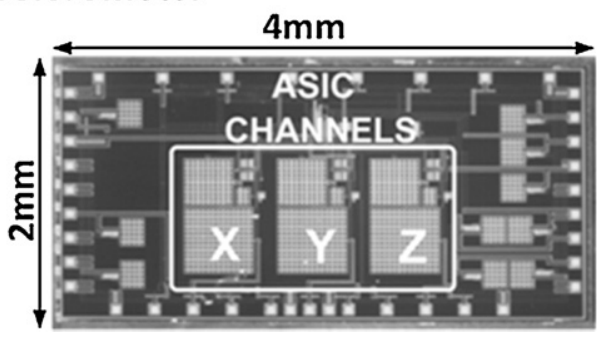

Fig. 5. Microphotograph of the accelerometer, strain sensor and readout ASIC chip.

\section{Readout architecture}

The readout architecture of the system has been described elsewhere [10]. Its main characteristics are summarized in the next paragraphs. The principle of operation is basically the one depicted in Fig. 6. When an acceleration force appears in the sensor, Fig. 6(a), the anti-phase actuation voltages applied to the capacitors shown in Fig. 6(b) are modulated. Current pulses flow in two directions, as shown in Fig. 6(c). At every rising edge the switches S1 and S2 are open while the INTEGRATE switch is closed (Fig. 7)
The current pulse is integrated in the feedback capacitor Cf, shown in Fig. 6(d). The system has several advantages that help to compensate problems related with the sensor such as offsets, nonlinearity, intrinsic gain, sensor's mismatch, etc. Varying the duty cycle of the actuation voltage, for example, can set the optimum SNR and gain for the sensors involved. Varying the amplitude of one of the excitation voltages with respect to the other can compensate for offsets or non-linearities. For the case of the strain sensor similar behavior is simulated with the only difference that the frequency of the signal is close to DC and the mass of the sensor is small.
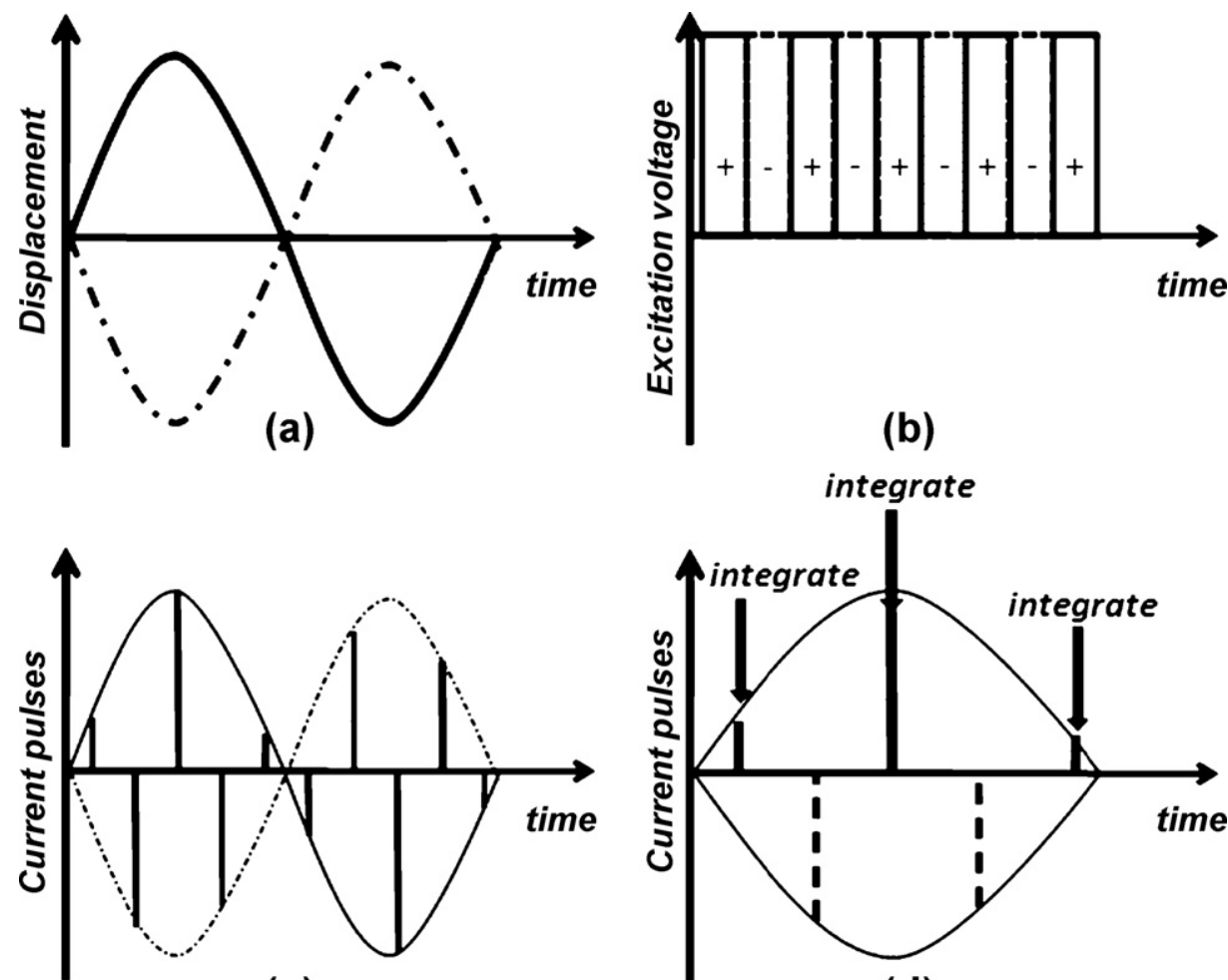

(c)

(b)

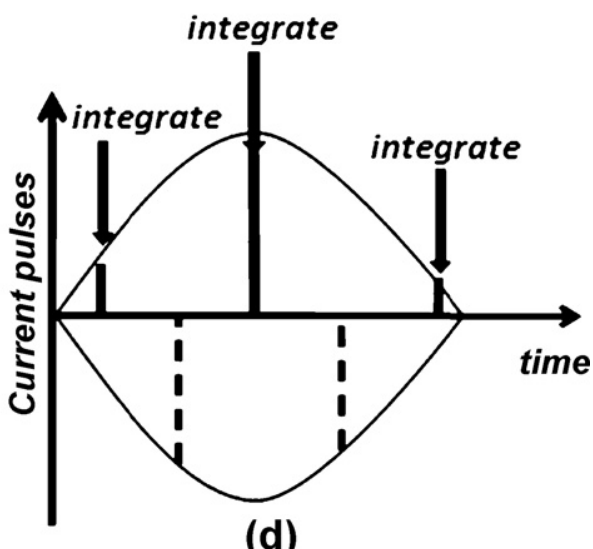

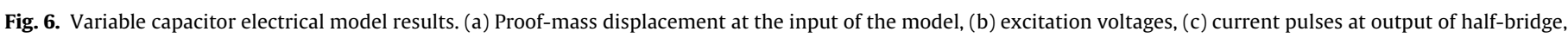
and (d) only positive pulses are integrated. 


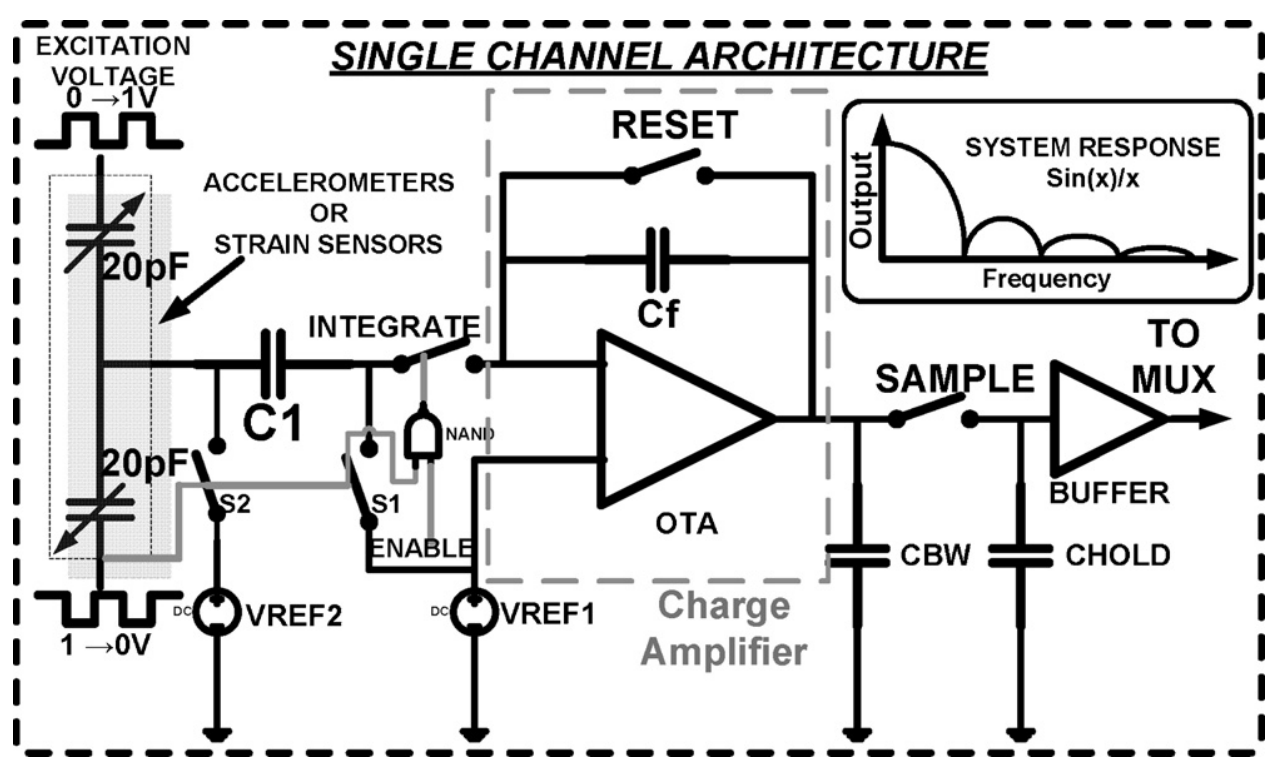

Fig. 7. Architecture of a single channel of the readout ASIC and $\sin (x) / x$ response of the system (inset).

Fig. 7 shows single channel architecture readout as well as the $\sin (x) / x$ system response. It differs from commonly used designs $[2,3]$ by decoupling the output of the sensor from the input of the charge amplifier (OTA) by a series capacitor C1 and switches connected to arbitrary variable reference voltages VREF1 and VREF2. The function of $\mathrm{C} 1$ is level shifting, to allow a different operating potential between the output of the sensors and the input of the OTA. In this way the excitation voltages of the sensor can be unipolar, while still allowing DC signal processing from the sensors signals. The architecture differs significantly from the modulation-demodulation approach, which is regularly used for similar devices and uses more power.

Architectures have been reported [1-4] in which linearity, resolution, bandwidth or gain is always matched to the particular type of device. In some designs the channel architecture is tailored to the properties of the sensor and, although some flexibility is added as shown in [7-9], its performance is not optimum $\left(\mathrm{FOM}=1.0 \times 10^{-18} \mathrm{~F} \sqrt{ }(\mathrm{W} / \mathrm{Hz})\right.$ ).

In this work the number of pulses $(N)$ can however be arbitrarily set depending on the desired SNR, bandwidth or gain. To first order these parameters are related by the following relationships: the gain is proportional with $N$, since all $N$ integrated pulses are added. The bandwidth is proportional to the inverse of $N$, since the sampling time is proportional to $N$ and the SNR is proportional to the square root of $N$ because gain is increasing with $N$ and noise is only increasing with square root of $N$. This is one of the key issues of the proposed architecture, it solves the issue of setting the gain of the system by increasing/decreasing the number of pulses instead of switching between integrating capacitors of different sizes. The readout gain has little temperature dependency, non-linearity and hysteresis, since the integrated capacitor Cf, the OTA performance and clock frequency are very stable.

The developed system has the added advantage that the same readout can be used to sense acceleration and strain with the purpose of reducing power consumption and making it more cost effective, there is no need to fabricate two different readouts to process the information coming from the two types of sensors. In principle the signals from the two types of sensors could be multiplexed at the input of the readout, thus using only one single channel to amplify their signals by alternating the reading of the sensors in a predetermined sequence. Nevertheless the accelerometer has to be always switched-on. One way to overcome this requirement is by operating the readout in a low power (lower bandwidth and SNR) mode and switched to high resolution acquisition mode in case of an earthquake event. The strain sensor is used for certain periods of time and a time-sharing scheme would make the decision to alternate between the accelerometer and the strain sensor without jeopardizing the data acquisition bandwidth and SNR immediate after an earthquake occurred.

\subsection{Measurements}

For the purpose of simulation of the whole system both sensors were modeled in a high level description language, namely VerilogA, as a pair of variable capacitors producing a $10 \% \Delta C$ full scale. The model allows the designer to accurately assess the gain, noise, sensitivity and motion artifacts within the circuit design environment with less computational effort. Results are in good agreement with simulations; the next paragraphs show the measurements.

Fig. 8 shows the measurements of the accelerometer sensor with the proposed readout. In the figure three channels are plotted corresponding to $X, Y$ and $Z$ directions and compared to commercially available accelerometers, namely PCB piezoelectric transducers model 393-B12 and 393-B31 [11]. The WL data corresponds to the sensors and readout design for this work whereas the B data correspond to the commercial devices. Both systems and obtained data comply with specifications for building integrity monitoring but the developed readout consumes less power than the commercial one.

As shown in [10] the resolution is $80 \mathrm{~dB}$ (13-bit) for vibration tones between 10 and $100 \mathrm{~Hz}$ and non-linearity $<1 \%$. The acceleration measurements are meant for recording the building's response during an earthquake event; the measured system noise floor (readout plus sensor) of $70 \mu \mathrm{g} / \sqrt{ } \mathrm{Hz}$ is well in line with the purpose of the application and does not degrade the expected performance of the system as shown in [13]. MEMS-based accelerometers like the ST Microelectronics LIS3L02AL have a $50 \mu \mathrm{g} / \sqrt{ } \mathrm{Hz}$ noise floor for a $\pm 2 \mathrm{~g}$ range [14] and the Crossbow CXL10TG3 a $20 \mu \mathrm{g} / \sqrt{ } \mathrm{Hz}$ for $\mathrm{a} \pm 2 \mathrm{~g}$ range [15] but their power consumption is in the order of $\mathrm{mW}$, smaller acceleration range and are larger in size, therefore not suitable for operation over extended periods of time without recharging or replacing batteries.

The readout is DC coupled therefore able to read very low frequency signals. This represents an advantage compared to standard 
Table 2

Power consumption at $V_{\mathrm{DD}}=1.6 \mathrm{~V}$.

\begin{tabular}{llll}
\hline Current & Power & Amplitude & Non-linearity \\
\hline $2.51 \mu \mathrm{A}$ & $4.00 \mu \mathrm{W}$ & $57.1 \mathrm{mV} / \mathrm{g}$ & $1.24 \%$ \\
$0.44 \mu \mathrm{A}$ & $0.70 \mu \mathrm{W}$ & $52.7 \mathrm{mV} / \mathrm{g}$ & $1.05 \%$ \\
$0.33 \mu \mathrm{A}$ & $0.52 \mu \mathrm{W}$ & $43.5 \mathrm{mV} / \mathrm{g}$ & $1.73 \%$ \\
$0.19 \mu \mathrm{A}$ & $0.30 \mu \mathrm{W}$ & $21.6 \mathrm{mV} / \mathrm{g}$ & $6.30 \%$ \\
\hline
\end{tabular}

modulation-demodulation schemes, which are always limited to a specific low frequency value, thus allowing for the readout of signals such as those from a strain sensor. Fig. 9 shows the results of strain sensor measurements when mounted on a test rebar.

Tests were taken over a time span of over $2000 \mathrm{~s}$ under continuous tensile strain and release to simulate the strain condition expected in the steel reinforcement of a concrete building during an earthquake action. The steel bar was taken beyond its elastic limit, corresponding to a load capacity of approximately $140 \mathrm{kN}$ and a yield strain of $2000 \mu \varepsilon$. The data were compared to commercially available systems compliant with the specifications. More specifically the reference sensors are resistive metal foil strain gauges HBM model LY41-3/700 [12]. Consistently with previous results [10], the test confirms that sensors and readout are able to measure over a range of $\pm 20,000 \mu \varepsilon$, which corresponds to the ultimate design strain condition of steel reinforcement under severe seismic action.

Fig. 10 shows the measurements of the sensitivity versus the integrated number of pulses $N$ for the accelerometer. As expected the sensitivity of the readout is proportional to the number of pulses $N$, while the integrated noise is proportional to $\sqrt{ } N$. The measurements were taken with a rate of 200 samples/s.

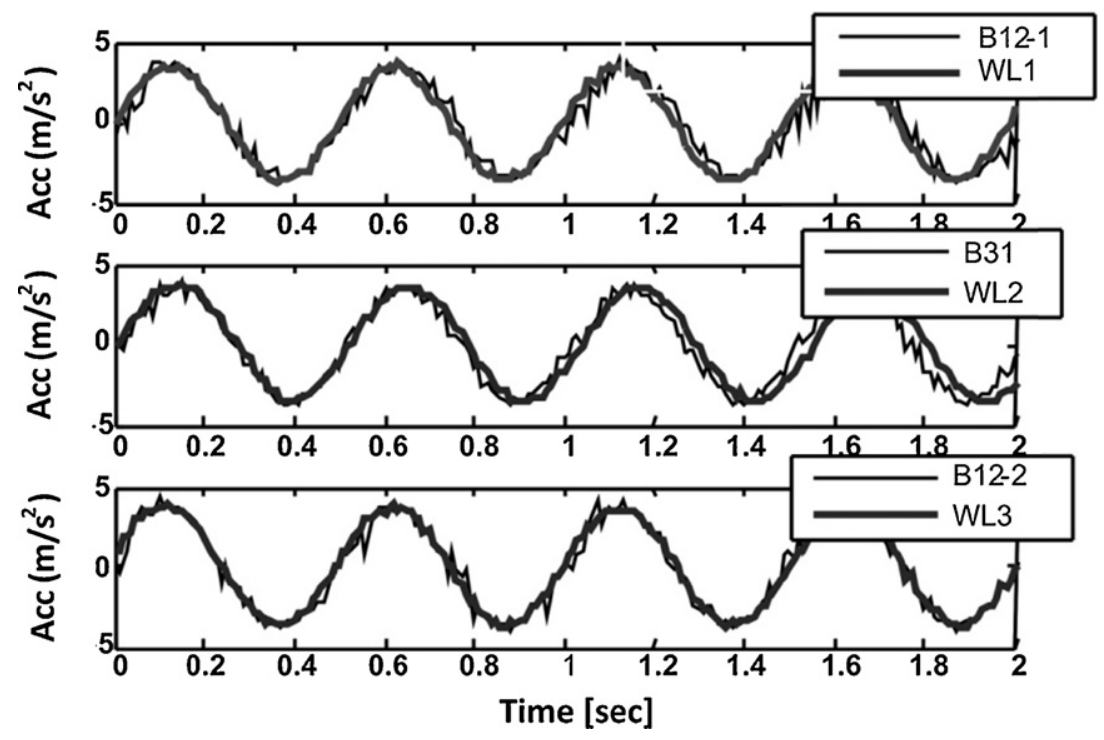

Fig. 8. Measured acceleration signals for $X, Y$ and $Z$ channels compared to commercial devices ( $N=24$ pulses).

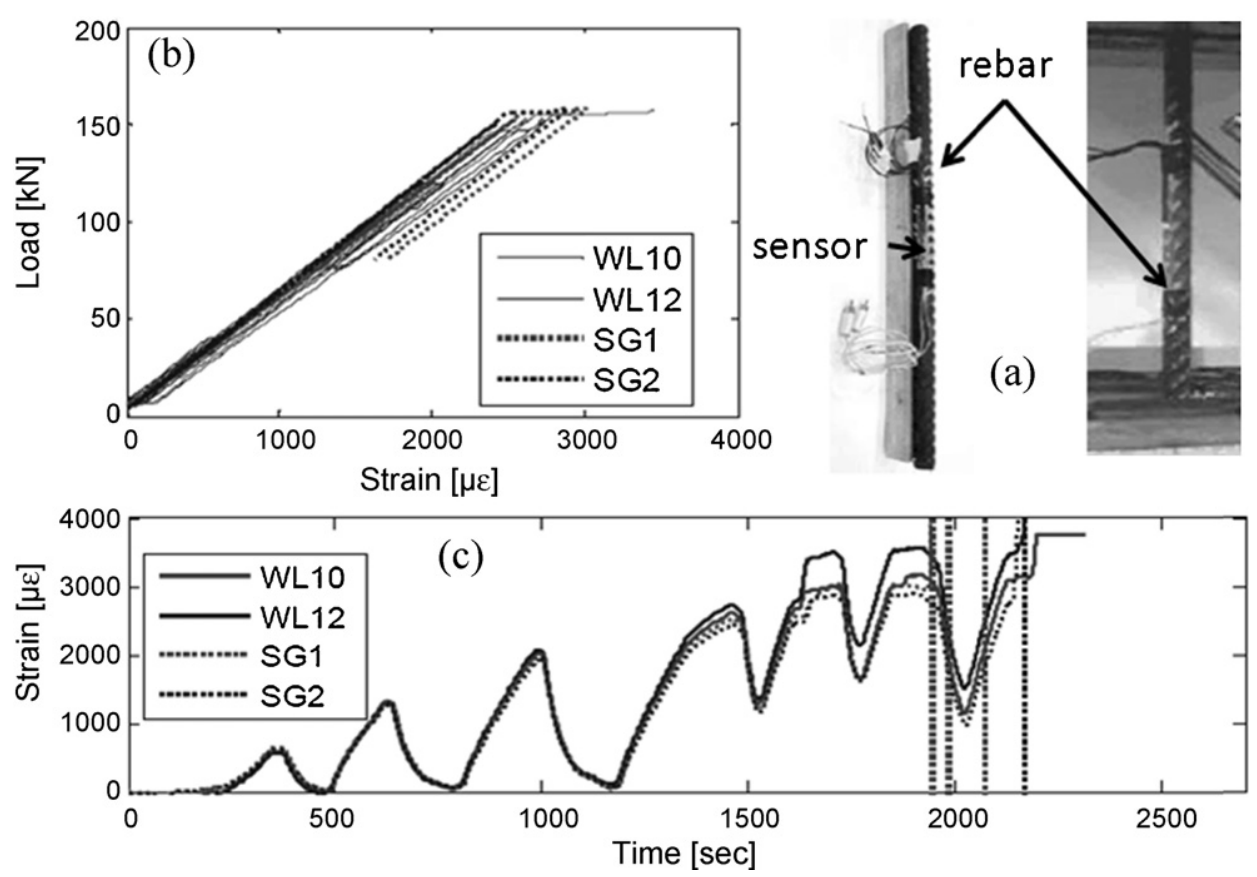

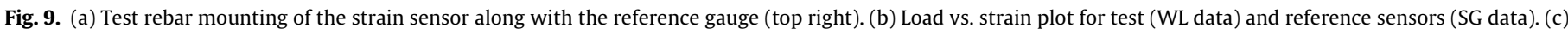
Strain vs. time history measurements. The position of the sensor in the rebar is also shown in the inset. 
Table 3

Comparison with state-of-the-art capacitive readouts.

\begin{tabular}{|c|c|c|c|}
\hline Parameter & Denison [5] & Paavola [6] & This work \\
\hline Accel/strain sensor range & $\sim \pm 1 \mathrm{~g}$ & $\pm 2 \mathrm{~g}$ & $\begin{array}{l} \pm 2.5 \mathrm{~g} \\
\pm 20,000 \mu \varepsilon\end{array}$ \\
\hline Supply voltage & $1.7-2.2 \mathrm{~V}$ & $1.0 \mathrm{~V}$ & $1.6 / 3.0 \mathrm{~V}$ \\
\hline Power & $1.5 \mu \mathrm{W}$ & $1.5 \mu \mathrm{W}$ & $440 \mathrm{nW} / 15 \mu \mathrm{W}$ \\
\hline Sensitivity & $125 \mathrm{mV} / \mathrm{g}$ & - & Variable \\
\hline Noise floor (acceler.) & $1 \mathrm{mg} / \sqrt{ } \mathrm{Hz}$ & $704 \mu \mathrm{g} / \sqrt{ } / \mathrm{Hz}$ & $70 \mu \mathrm{g} / \sqrt{ } / \mathrm{Hz}$ \\
\hline Non-linearity & $<1 \%$ & - & $\begin{array}{l}<1 \% \text { (accel.) } \\
<0.6 \% \text { (strain sensor) }\end{array}$ \\
\hline Bandwidth & $10 \mathrm{~Hz}$ & $1 \mathrm{~Hz}$ & $100 \mathrm{~Hz}$ \\
\hline Figure-of-Merit & $8.1 \times 10^{-22} \mathrm{~F} \sqrt{ }(\mathrm{W} / \mathrm{Hz})$ & $1400 \mu \mathrm{W} \mu \mathrm{g} / \mathrm{Hz}$ & $\begin{array}{l}4.41 \times 10^{-20} \mathrm{~F} \sqrt{ }(\mathrm{W} / \mathrm{Hz}) \\
881 \mu \mathrm{W} \mu \mathrm{g} / \mathrm{Hz}\end{array}$ \\
\hline Technology & $0.8 \mu \mathrm{m}$ CMOS & $0.25 \mu \mathrm{m}$ CMOS & $0.25 \mu \mathrm{m}$ CMOS \\
\hline Active area & - & $2.25 \mathrm{~mm}^{2}$ & $2 \mathrm{~mm}^{2}$ \\
\hline
\end{tabular}

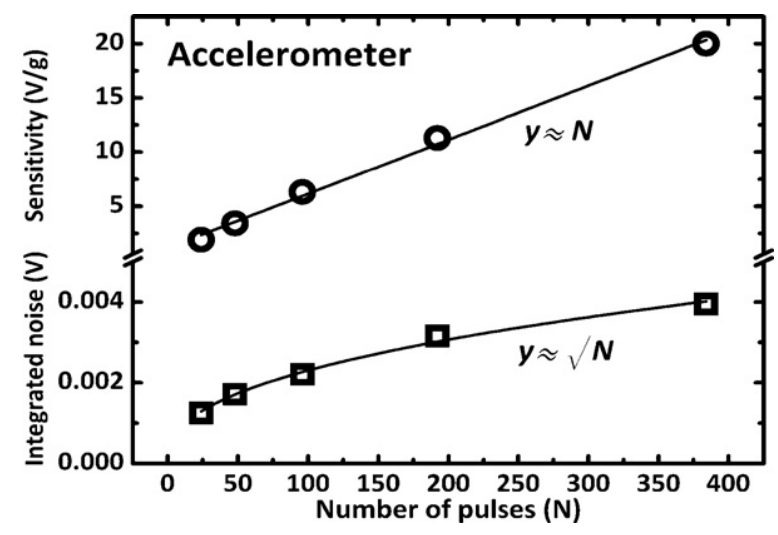

Fig. 10. Measured sensitivity and total integrated noise for the accelerometer vs. number of pulses $N$.

The sensitivity and range of acceleration (or strain) can be set according to the device and application specifications. Alternative methods have been reported elsewhere; the settings of reconfigurable building blocks create an adaptable generic architecture for a wide range of capacitive sensors [9]. Nevertheless power consumption remains high and not adequate for low power applications. In this work the readout was designed to optimize power consumption. Previous results shown in [10], with $V_{\mathrm{DD}}=3.0 \mathrm{~V}$ show a power consumption of $15 \mu \mathrm{W}$. With a bias voltage of $1.6 \mathrm{~V}$, improved levels of power consumption for the readout circuit can be reached as shown in Table 2.

The amplitude of the signal decreases by a factor of 10 but the power consumption decreases by a factor of 100 while keeping the rest of the parameters as in the case of $3.0 \mathrm{~V}$. The results in the table show also the non-linearity values which are still within specifications. At the minimum power consumption of $330 \mathrm{nW}$ the non-linearity increases to $6.30 \%$ which is the extreme case of the operating range of the sensor system. The experimental results are shown in Fig. 11.

In spite of the amplitude of the signal being lower the system can compensate by integrating more pulses, as mentioned in previous paragraphs, and therefore restore the Dynamic Range to full railto-rail. The system response time will decrease but for the target applications this does not represent a major problem.

\section{Conclusions}

Results show that the readout architecture can work with Ultra-Lower-Power consumption while keeping linearity under the expected performance. Battery lifetime is then extended to several years' operation. Compared to the figures of merit proposed in $[5,6]$ this work has $4.41 \times 10^{-20} \mathrm{~F} \sqrt{ }(\mathrm{W} / \mathrm{Hz})$ and $881 \mu \mathrm{W} \mu \mathrm{g} / \mathrm{Hz}$,

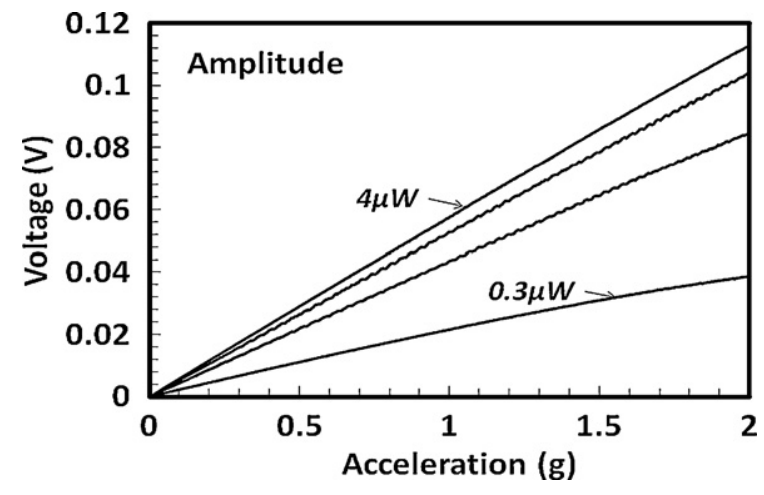

Fig. 11. Signal amplitude at $1.6 \mathrm{~V}$ for several bias current values.

respectively. The proposed architecture has the lowest reported equivalent acceleration noise level, highest bandwidth and has the unique advantage of offering tradeoff between SNR, bandwidth and power. When compared to commercial accelerometers and strain sensors the readout offers the user competitive specifications. The design was fabricated on TSMC $0.25 \mu \mathrm{m}$ CMOS with M-i-M capacitors. The total power consumption of the 3 channels is $15 \mu \mathrm{W}$. The clock and excitation voltages for the sensors are external. Recent measurements show that the architecture can work with less power consumption $(0.52 \mu \mathrm{W})$ still within the expected range of acceleration and strain (Table 1 ).

Table 3 shows the benchmarking of the proposed architecture. No similar system has been reported which handles both accelerometers and strain sensors with a minimum of power consumption, low noise floor, variable sensitivity and larger bandwidth. Most of the systems reported are custom-made systems for a particular sensor (which usually has a large sensitivity in a narrow band) with low-power consumption. Also shown in Table 3 is the lowest power consumption measurement reported.

The presented system can deal with different sensors without jeopardizing the power consumption, important in building integrity monitoring which requires battery operated systems for several years. It also represents a very cost effective solution since the same readout can be used for accelerometers or strain sensors, making the integration of the final monitoring system simpler and cheaper.

Flexibility is also a major asset for the system, sensors with different sensitivities, offsets and mismatch can be easily readout modifying the timing and duty cycle of the excitation pulses and the number of integrated ones. A single readout system for both accelerometers and strain sensors makes the system more cost effective and power saving. 


\section{Acknowledgments}

This work is part of the Human++ Program of imec-Holst Centre, The Netherlands, and partially funded by the MEMSCON project (EC Seventh Framework Programme Grant Agreement n. CP-TP 212004-2).

\section{References}

[1] M. Suster, et al., A high performance MEMS capacitive strain sensing system, Journal of Microelectromechanical Systems 15 (5) (2006) 1069-1077.

[2] C. Yi-Chen, Linear Programmable Switch-Capacitance Gain amplifier, US Patent No. 2008/0297243, December 4, 2008

[3] M. Weng, et al., A high-speed low-noise CMOS 16-channel charge-sensitive preamplifier ASIC for APD-based PET detectors, IEEE Transactions on Nuclear Science 5 (4) (2005) 898-902.

[4] ADXL330 Datasheet, Small, Low Power, 3-Axis $\pm 3 \mathrm{~g}$ iMEMS Accelerometer, Analog Devices, Inc., 2007.

[5] T. Denison, et al., A $2 \mu \mathrm{W}$ three-axis MEMS-based accelerometer, in: Instrumentation and Measurement Technology Conference - IMTC 2007, Warsaw, Poland, May 1-3, 2007.

[6] M. Paavola, et al., A micropower $\Delta \Sigma$-based interface ASIC for a capacitive 3axis micro-accelerometer, IEEE Journal of Solid-State Circuits 44 (11) (2009) 3193-3210.

[7] B. Boser, T. Howe, Surface micromachined accelerometers, IEEE Journal of Solid State Circuits 31 (March (3)) (1996) 366-375.

[8] S.R. Hunter, Low Noise Radiation Sensor, US Patent No. 2008/0122453, May 29, 2008.

[9] W. Bracke, et al., Generic architectures and design methods for autonomous sensors, Sensors and Actuators A: Physical 135 (2) (2007) 881-888.

[10] J. Santana, et al., A 3-axis accelerometer and strain sensor system for building integrity monitoring, in: Proceedings of the Transducers 2011 Conference, Beijing, China, 2011, pp. 36-39.

[11] http://www.pcb.com/spec_sheet.asp? model=393C

[12] http://www.disensors.com/downloads/products/Y\%20Series\%20Strain \%20Gauges_333.pdf.

[13] A. Amditis, et al., Wireless sensor network for seismic evaluation of concrete buildings, in: Proc. 5th European Workshop on Structural Health Monitoring (EWSHM 2010), Sorrento, Italy, 29 June-02 July, 2010.

[14] http://datasheet.octopart.com/LIS3L02AL-STMicroelectronics-datasheet603974.pdf

[15] http://pdf.directindustry.com/pdf/crossbow/the-tg-seriesaccelerometers/19299-72237-_2.html.

\section{Biographies}

Juan Santana obtained his PhD in Semiconductor Devices from the University of Lancaster, U.K. and in 1992 joined the engineering department. In 1997 he joined CINVESTAV, a research centre in Mexico. From 1998 to 2001 he directed the Semiconductor Technology Centre (CTS) of CINVESTAV. He worked as a commissioned engineer in several ASIC projects for automotive applications for ATMEL Corp., in Columbia, Maryland. In 2001 he joined the Motorola Center for Semiconductor Technology. Since 2006 he works for Holst Centre, The Netherlands. He has published more than 30 papers in international journals, supervised several M.Sc. dissertations and holds one patent.

Richard van den Hoven studied Electronics at the Technical University in Eindhoven, where he graduated in 2006 at the group Mixed Signal Micro Electronics on "A pre-correction method for improved static linearity using parallel DACs". In
2006 he joined Philips Semiconductors (NXP) in Eindhoven, the Netherlands. Since 2007 he works for Holst Centre in the Netherlands, on ASIC design of Analog to Digital conversion and capacitive readout. He has published two papers and holds one patent.

Chris van Liempd is a Senior Researcher at Holst Centre since 2009. He has been system architect at Philips Semiconductors, NXP and Sunext. In 1998 he became part of the Philips Optical Storage group and played a major role in several projects in this group. He has more than 30 years experience in analog electronics engineering. He is currently working on DC-DC and AC-DC converters for power management and energy harvesting. He has published several papers and holds patents.

Mikael Colin received a Postgraduate degree in Optics in 2000 from the University of Saint-Etienne, France. He subsequently joined Bookham Technologies, UK (2000), and Opsitech, France (2002), as an R\&D engineer where he mainly worked in designing integrated components for Optical Networks. In 2004, he moved to Memscap, France, as a MEMS designer. His work mainly focused on the design of inertial components for Military, Space, Biomedical and Industrial applications and on Energy scavenging solutions using the MEMS technology. Since 2011, he works on the development of implantable energy scavengers at the TIMA laboratory, France.

Nicolas Saillen is a MEMS Product Engineer with a M.Sc. degree in Micro Systems Engineering from EPFL, Lausanne. He has 5 years experience in MST process, product development, and international project management at Thermo Fisher Scientific (previously $\mathrm{C} 2 \mathrm{~V}$ ). His work involves, among other things, the development of micro components such as: a flow sensor, force sensor, a piezo driven inkjet nozzle, and fluidic valves.

Daniele Zonta achieved his Doctorate in Structural Mechanics at the University of Bologna in 2000. He worked as a Post-Doctoral researcher at the University of California, San Diego, in the period 2000-2001. He has been visiting scholar at the University of Michigan (2010) and at Princeton University (2009 and 2011). Since 2001, he is Assistant Professor of Structural Engineering at the University of Trento teaching precast concrete bridge design and smart structures. His research activity includes: bridge management; Bayesian decision-making, structural health monitoring; fiber optics and smart sensor technologies; all as applied to civil structures. $\mathrm{He}$ is author of over one hundred technical publications.

Davide Trapani graduated in 2010 in civil engineering at the University of Trento. He is currently Research Assistant in the Intelligent Infrastructure group at the same University working on the laboratory validation of smart sensor technologies. He authored four technical papers.

Tom Torfs received the degree of Industrial Engineer in Electronics, specialization Design Technology in 2001 from De Nayer Institute, Belgium. He is since 2001 a systems design engineer in the sensor electronics group at IMEC. He has designed a range of low power wireless sensor modules, with a focus on biopotential acquisition systems for body area networks and autonomous systems. He has also been active in developing demonstrators using IMEC's 3D and 2D integration technology as well as participating in the design of a 3D modular needle array for in vivo applications (Neuroprobes EU project).

Chris Van Hoof is Program Director of More than Moore SIP Systems at IMEC in Leuven. He is also Associate Department Director of Interconnect, Packaging and System Integration. He received a $\mathrm{PhD}$ in Electrical Engineering from the University of Leuven in 1992. At IMEC, he became successively head of the detector systems group, Director of the Microsystems Department and Integrated Systems Department, and Program Director. His research concerns autonomous sensor nodes focusing on advanced packaging and interconnect technology and system integration. Since 2000 he is a professor at the University of Leuven advising 12 doctoral students. 